E-Review 1-2013

Bologna (BraDypUS) 2014

\#usopubblico

ISSN: 2282-4979

ISBN: $978-88-98392-05-6$

DOI:

pp. 267-272

\title{
Il patrimonio scomodo del Novecento europeo nel progetto Atruim a Forlì
}

Il Novecento ha lasciato tracce difficili nelle città d'Europa. I regimi che si sono succeduti hanno trasformato urbanisticamente e architettonicamente l'intero territorio e oggi è complesso relazionarsi con questi segni. Il progetto europeo Atruim - Architecture of Totalitarian Regimes in Urban Managements si propone di valorizzare, con attenta cura e senza intenti mistificatori, proprio il "patrimonio scomodo" di 11 paesi dell'Europa del sud.

The XX Century left difficult traces inside the european cities. The different totalitarian regimes transformed the entire urban form and architectural style: now, the relationship with these marks is very complex. The european project Atrium - Architecture of Totalitarian Regimes in Urban Managements wants to give careful attention to this "inconvenient heritage" of 11 countries of the South-east Europe, without any falsification.

\section{Architetture imbarazzanti}

Il Novecento ha lasciato tracce difficili nelle città d'Europa. I regimi che si sono succeduti hanno trasformato urbanisticamente e architettonicamente l'intero territorio europeo. Non che ciò non fosse accaduto prima, anzi, è una naturale inclinazione delle forme di potere quella di dare la propria impronta alle città, a fini celebrativi, propagandistici o di controllo sociale.

Tuttavia, per le possibilità date dalle tecniche costruttive e le necessità derivate dall'incremento della popolazione, nel Novecento questo fenomeno si è dato in proporzioni impressionanti e in tempi ridotti. 
Per gli abitanti, oggi, non è sempre facile relazionarsi con questi edifici o interi quartieri, addirittura intere città: l'uso di queste strutture avviene a volte in continuità con la funzione per cui sono state progettate, a volte secondo paradossali contro-usi. In alcuni casi la consapevolezza della natura di questi edifici porta al loro abbandono: è l'imbarazzo sollevato da queste tracce a renderne impossibile una qualsiasi riappropriazione.

In area tedesca questo problema si è reso evidente immediatamente dopo la fine del secondo conflitto mondiale: i grandi edifici di rappresentanza progettati dai nazisti proprio per lasciare una traccia ieratica e solenne ai posteri, vennero demoliti, laddove possibile, non tanto per problemi di statica, ma molto probabilmente per evitare che adempissero alla propria funzione. Albert Speer racconta nelle sue Memorie come Hitler avesse scelto granito e mattoni per le proprie Wunderwerke, "opere meravigliose" come le definiva il führer, perché "viste come rovina" avrebbero potuto eternare il Reich allo stesso modo in cui il Colosseo faceva per l'antica Roma.

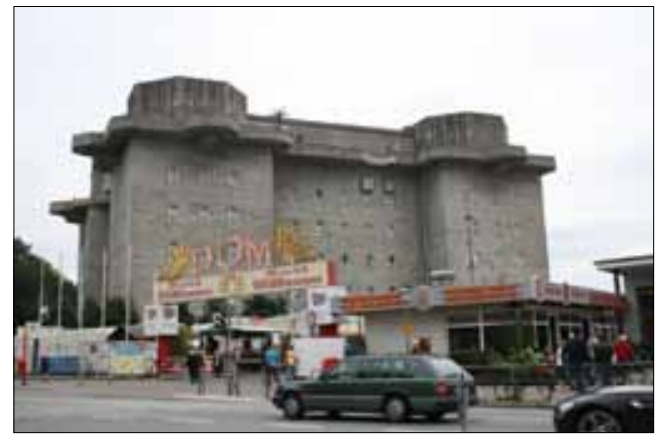

Flaktürm di Amburgo

Tuttavia, a differenza di quanto si possa pensare, sono state effettivamente realizzate, e ci restano oggi, diverse architetture progettate dal nazismo: oltre al famoso Reichsparteitagsgelände di Norimberga - composto da una Kongresshalle in forma di Colosseo, lo Zeppelinfeld e la Grosse Strasse lastricata di granito -, accanto ai grandi sistemi di bunker (le linee difensive come l'Atlantikwall o le torri della contraerea, le cosiddette Flaktürme), esistono complessi come le Ordensburgen (fortezze militari per la formazione) o i Thingstätten, teatri all'aperto per mettere in scena testi sull'epica di fondazione del movimento.

In questi ultimi casi, nel dopoguerra le strutture sono state riutilizzate assecondando le loro funzioni militari e teatrali, confidando che il cambiamento di segno politico bastasse a neutralizzarne il significato originario.

Tuttavia, il messaggio che emana dal gigantismo esasperato che caratterizza buona parte dei progetti nazisti, è molto difficile da controllare: l'unico modo per farlo passa attraverso la consapevole accettazione del loro significato, del motivo per cui sono sorte, comunicandolo senza ambigue operazioni di nascondimento, dimenticanza o mistificazione. 
In quest'estate 2013, infatti, se a Norimberga si discute sugli ingenti stanziamenti per il restauro degli edifici del Reichsparteitagsgelände, molto meno si riflette sulle operazioni di riappropriazione della Seebad Prora, una colossale colonia marina progettata per ospitare 20.000 persone nella baia più bella dell'isola di Rügen. Senza avere mai assolto

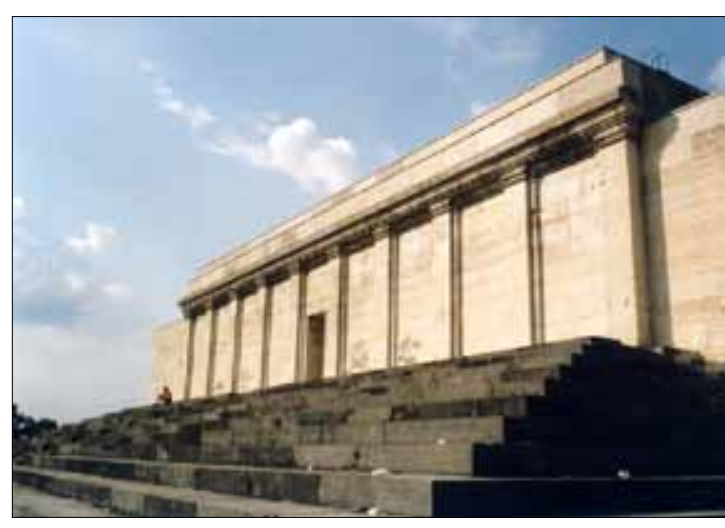

Tribuna dello Zeppelinfeld, Reichsparteitagsgelände di Norimberga alla funzione prevista durante gli anni del Terzo Reich - quando ospitò sfollati durante il conflitto - l'interminabile edificio, dallo sviluppo lineare pari a circa 4,5 chilometri, venne usato prima dall'armata rossa poi dalla Nationale Volksarmee, in questo secondo caso anche assecondando la sua vocazione turistica. Dopo il 1990 alcune porzioni sono state utilizzate per esposizioni storiche, altre come ostello. Quest'anno, il progetto Neues Prora, condotto da un privato, si propone di trasformare un blocco di Prora in residenze balneari di lusso, senza specificare, nel materiale informativo pubblicitario, per quale motivo e da chi il complesso fosse stato edificato a partire dal 1936.

\section{The Architecture of Totalitarian Regimes in Urban Manage- ments: un progetto per l'Europa sud orientale}

Proprio questo «rapporto complesso, contraddittorio e talvolta scomodo con alcuni periodi della storia europea», e in particolare con il patrimonio architettonico che quei periodi hanno lasciato, è stato al centro del progetto europeo Atrium - Architecture of Totalitarian Regimes in Urban Managements, che ha coinvolto 18 partner di 11 paesi (Italia, Albania, Bosnia-Erzegovina, Bulgaria, Croazia, Grecia, Romania, Slovacchia, Slovenia, Serbia, Ungheria) tra il 2010 e il 2013, avendo come capofila il comune di Forlì.

Se nel caso forlivese (e di tutto il territorio della provincia di Forlì-Cesena) si tratta di architetture razionaliste del Ventennio fascista, per i numerosi partner dell'Europa sud orientale, il patrimonio "scomodo" riguarda prevalentemente i progetti architettonici dei regimi comunisti, quindi realizzati nell'arco di tempo che va dagli anni Cinquanta agli anni Settanta. 
Diversi nello stile oltre che nel riferimento politico, questi progetti hanno tuttavia degli elementi in comune: la committenza statale, soprattutto di grandi opere, realizzate attraverso un processo decisionale imposto dall'alto e volto a suscitare consenso; la pervasività del regime autoritario, che si inserisce nella vita quotidiana della società, plasmandone gli spazi (scuole, colonie, istituzioni, servizi). Il progetto di valorizzazione di questo patrimonio scomodo passa attraverso la necessaria consapevolezza del peso del passato che lo grava: non vi è alcun intento assolutorio o, peggio, revisionista. Si tratta anzi di sollevare lo sguardo e accettare come quello che ora viene considerato un passato terribile abbia avuto un lungo presente di splendore e consenso, di cui quelle tracce sono testimonianza. Spesso, inoltre, la reazione più comune per mettere in atto una presa di distacco da quei periodi politici è bollarne i progetti architettonici come "brutti". In realtà, se pensiamo al caso del razionalismo italiano, ovvero la corrente che più ha inverato i progetti del fascismo, si tratta in molti casi di progetti dal disegno raffinato, legati al più ampio alveo del modernismo che, ad esempio nella vicina Germania era invece espressione di una committenza politica di segno opposto come quella della Repubblica di Weimar. Il nazismo si avvalse del funzionalismo solo per alcuni progetti, come quelli delle strutture industriali, non certo per gli edifici di rappresentanza, per i quali scelse un neoclassicismo ispirato all'eternità della potenza romana, ma reso ipertrofico da un gigantismo programmatico.

\section{Una nuova trans-national cultural route}

L'obiettivo del progetto Atrium è stato l'avvio per un processo di creazione di una "rotta culturale transnazionale" che possa valorizzare - a livello culturale ma anche turistico e, quindi, economico - questi luoghi, queste tracce, questi edifici di cui è disseminata l'Europa sud-orientale, facendo in modo che divengano spunto per una presa d'atto consapevole del loro significato, sostituendo quell'atteggiamento di giudizio, ma allo stesso tempo di rimozione, che abitualmente li circonda.

Attualmente esistono quasi una trentina di trans-national cultural routes promosse dal Consiglio d'Europa, ma riguardano per lo più le vie dei pellegrinaggio (la via Francigena, il cammino di Santiago), epoche remote come l'età del ferro, oppure la medioevale via Regia, i luoghi dell'eredità ebraica o dell'Andalusia musulmana: la rotta riferita ai tempi più recenti sembra essere quella sui luoghi mozartiani, tra Salisburgo e Milano.

Chiuso il Novecento, resta il suo grave carico storico, fatto di due guerre mondiali - ma profondamente e dolorosamente europee - e dei regimi che le segui- 
rono: una trans-national cultural route che passi attraverso il paesaggio urbano lasciato dai regimi del Novecento potrebbe aiutare i cittadini europei non solo a esplorare il proprio passato, ma a comprendere le stratificate ferite da cui sta nascendo un'Europa unita.

Tra il 2010 e il 2013 all'interno di Atrium sono state portate avanti numerose iniziative di confronto e

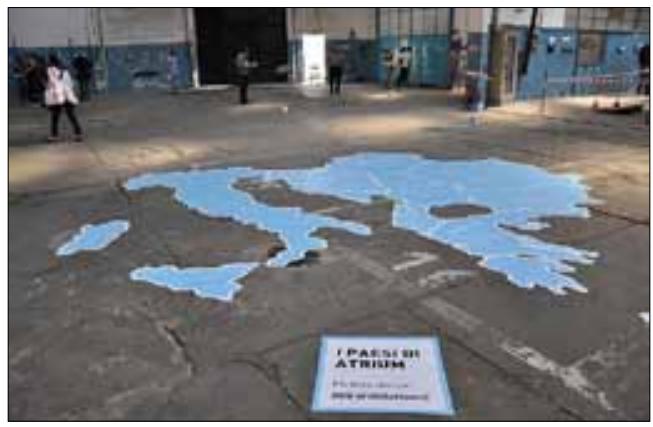

Presentazione progetto Atrium, Forlì giugno 2013, foto di Luca Cagnazzo di ricerca, per poter valutare le opportunità di restauro e valorizzazione di questo patrimonio difficile: sono stati individuati luoghi ed edifici, esaminando il loro rilievo storico-architettonico e la criticità di eventuali interventi di recupero; inoltre, sono stati raccolti e catalogati archivi fotografici e altre testimonianze utili a creare anche un bacino di informazioni per un'ulteriore approfondimento dei diversi casi e del tema in generale. Inoltre, attraverso mostre e convegni, realizzati collaborando anche con altre realtà presenti sul territorio, il progetto di candidatura è stato presentato anche al pubblico di cittadini, turisti e studenti.

A metà giugno 2013, alla chiusura del progetto, si è arrivati alla costituzione dell'Associazione Atrium, titolare della presentazione formale della candidatura della rotta al Consiglio d'Europa: la sede sarà proprio a Forlì, presso l'Ex GIL di viale della Libertà, presidente è stato eletto l'assessore alla cultura della città, Patrick Leech, mentre vice presidente sarà Irina Belušić Maggi, rappresentante del comune croato di Labin.

In particolare, tra le proposte attivate da Atrium, la mostra Totally Lost presentava una campagna fotografica organizzata dall'Associazione "Spazi Indecisi" coinvolgendo fotografi tramite una open call in rete: sono arrivate 2.300 immagini da 11 paesi focalizzate sul patrimonio "totalitario" in abbandono, quello che per una sorta di "malattia dello sguardo" si tende a fare

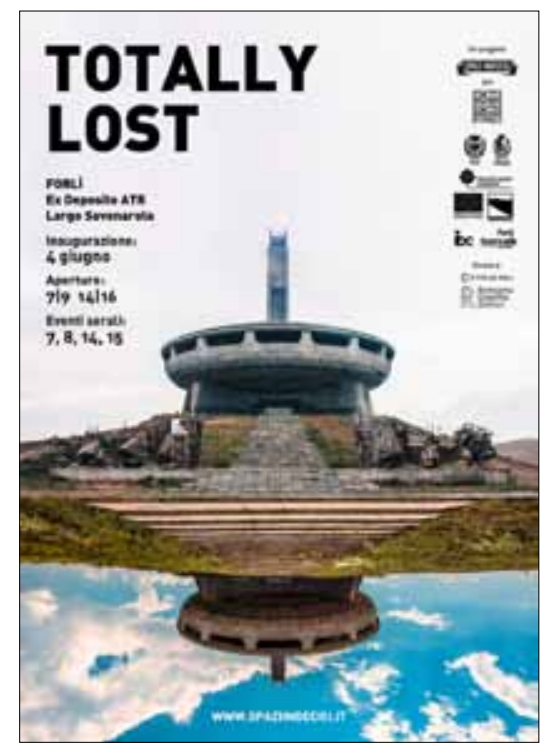

Locandina della mostra Totally lost 
sparire dalla propria vista anche quando per proporzioni, forme, collocazioni paesaggistiche, non è proprio possibile non vedere. È il timore che possa riproporsi che non ci permette di sostenere la vista sui resti del nostro passato più prossimo: perché parla di qualcosa a noi ancora troppo vicino o addirittura interno.

Alle soglie del centenario della Grande guerra, tuttavia, colpisce come non esista una trans-national european cultural route dedicata a quell'evento, terribile e seminale per la storia dell'Europa. Forse anche quel lutto non è stato ancora elaborato a livello transnazionale.

\section{Risorse}

Centro di documentazione Prora http://www.proradok.de

Progetto europeo Atrium http://www.atrium-see.eu/ita

Atrium Forlì http://atrium.comune.forli.fc.it

Pagina facebook Atrium Forlì http://www.facebook.com/atriumforli

Totally Lost / spazi indecisi http://www.spaziindecisi.it

Cultural Routes of the Council of Europe http://www.coe.int/t/dg4/cultureheritage/culture/routes/default_en.asp 